\title{
A 1-4 GHz DLL Based Low-Jitter Multi-Phase Clock Generator for Low-Band Ultra-Wideband Application
}

\author{
Tsung-Te Liu and Chorng-Kuang Wang \\ Graduate Institute of Electronics Engineering, and Department of Electrical Engineering, \\ National Taiwan University, 106 Taipei, Taiwan R. O. C. \\ E-mail: ckwang@cc.ee.ntu.edu.tw
}

\begin{abstract}
This paper presents the implementation of a low-jitter system clock generator for low-band ultra-wideband (UWB) application based on a wide-range adaptive-bandwidth delay-locked loop (DLL). The false-locking problem commonly along with the wide-range DLL is eliminated by the proposed digital selfcorrecting loop which also speeds up the lock-in time of the DLL. With self-biased techniques, the proposed DLL adaptively adjusts bandwidth and exhibits optimal jitter transfer characteristic over a wide frequency range and across process, voltage, and temperature (PVT) variations. Fabricated in a 0.18$\mu \mathrm{m}$ CMOS technology, the design achieves an output multiphase sampling clock rate of 1 to $4 \mathrm{GHz}$ and exhibits the maximum input tracking jitter of $12.06 \mathrm{ps}(\mathrm{rms})$ and $88.9 \mathrm{ps}$ (pkpk) over the operating frequency range from 31.25 to $125 \mathrm{MHz}$. The prototype occupies an active area of $360 \times 245 \mu \mathrm{m}^{2}$ and consumes $32 \mathrm{~mW}$ from a $1.8-\mathrm{V}$ supply at $125 \mathrm{MHz}$.
\end{abstract}

\section{INTRODUCTION}

The Federal Communications Commission (FCC) has opened the frequency bands for unlicensed ultra-wideband (UWB) wireless systems since February, 2002 [1]. System architectures for pulse-based UWB radio have been recently proposed targeting applications such as positioning system and sensor network. One critical aspect of VLSI implementation of pulsebased UWB radio is the accurate generation of timing signals to support reliable data communications [2].

Phase-locked loops (PLLs) and delay-locked loops (DLLs) have been typically employed to generate high-frequency accurate system clock from a low-frequency, precise, and stable oscillator. Usually, DLLs are easier than PLLs to implement the clock generator. A DLL is unconditionally stable and only needs one capacitor in its first-order loop filter, while a PLL generally requires a higher order filter which usually occupies large areas or needs to be off chip in order to guarantee the stability and performance. Moreover, a DLL offers better jitter performance than a PLL because phase errors induced by supply or substrate noises do not accumulate over many cycles in the voltagecontrolled delay line (VCDL) [3]. However, conventional DLLs may suffer from false locking, which is undesirable if multiphase clocks are needed. The maximum and the minimum delays of the VCDL, $T_{\mathrm{VCDL} \cdot \max }$ and $T_{\mathrm{VCDL} \cdot \min }$, should satisfy the following inequalities to avoid a false locking [4]:

$$
\begin{aligned}
0.5 \times T_{\mathrm{CLK}} & <T_{\mathrm{VCDL} \cdot \min }<T_{\mathrm{CLK}} \\
T_{\mathrm{CLK}} & <T_{\mathrm{VCDL} \cdot \max }<1.5 \times T_{\mathrm{CLK}}
\end{aligned}
$$

\footnotetext{
This work was supported in part by MediaTek Inc. Hsin Chu, Taiwan 300
}

or, equivalently, in terms of $T_{\mathrm{CLK}}$ :

$$
\begin{aligned}
\operatorname{Max}\left(T_{\mathrm{VCDL} \cdot \min }, 2 / 3\right. & \left.\times T_{\mathrm{VCDL} \cdot \max }\right)<T_{\mathrm{CLK}} \\
& <\operatorname{Min}\left(2 \times T_{\mathrm{VCDL} \cdot \min }, T_{\mathrm{VCDL} \cdot \max }\right)
\end{aligned}
$$

where $T_{\mathrm{CLK}}$ is the period of the input reference clock. If the target clock period satisfies the inequality (2), the DLL works and does not posses a false-locking. However, if $T_{\mathrm{VCDL} \cdot \max } \geq 3 \times$ $T_{\mathrm{VCDL}}$ min, which is usually the case when process, voltage, and temperature (PVT) variations are taken into consideration, then there is no range of $T_{\mathrm{CLK}}$ that satisfies the inequality (2). Therefore, the conventional DLL can only operate over a narrow frequency range if an exotic tedious trimming circuit for correct locking is not employed [4].

This paper proposes a novel DLL architecture without the false-locking problem, which combines two techniques [5], [6] for implementation of a low-jitter wide-range multi-phase clock generator. The output sampling clock rate of the proposed DLL spans from $1 \mathrm{GHz}$ to $4 \mathrm{GHz}$, which is sufficient for low-band $960 \mathrm{MHz}$ UWB system application [2].

The rest of this paper is organized as follows. Section II describes the proposed DLL architecture followed by the circuit design in Section III. Experiment results are presented in Section IV. Section V concludes this paper.

\section{DLL ARCHITECTURE}

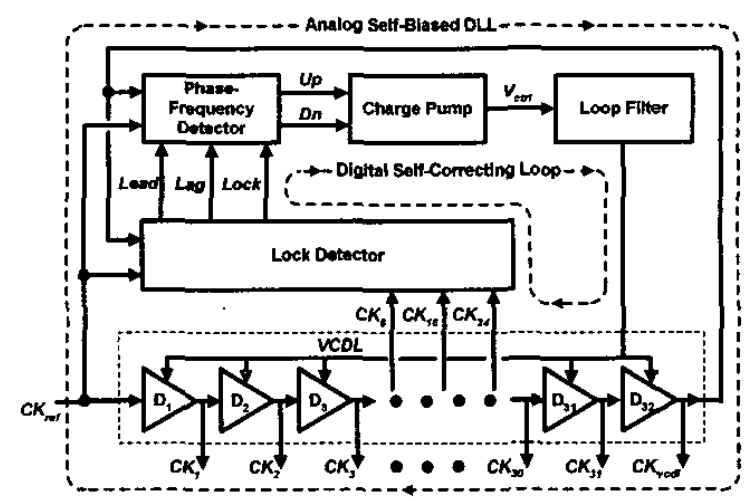

Fig. 1 Block diagram of the proposed DLL architecture.

Fig. 1 shows the architecture of the proposed low-jitter widerange adaptive bandwidth DLL, which consists of a main analog self-biased DLL and an auxiliary digital self-correcting loop. The phase-frequency detector (PFD), charge pump, loop filter, and 32-stage VCDL compose the DLL core. The self-biased circuits adjust the DLL loop bandwidth adaptively. Therefore, 
the loop stability and an optimal jitter transfer characteristic can be maintained over a wide frequency range and across PVT variations [6]. The auxiliary digital self-correcting loop consists of the same components of the DLL core except employing a lock detector to replace the PFD. It continuously monitors the DLL acquisition behavior and takes control of the loop if the phenomenon of false locking is detected. The lock detector utilizes three additional delayed clocks, $C K_{8}, C K_{16}$, and $C K_{24}$ from VCDL to determine the preset lock-in state. It then generates three digital control signals, namely, Lead, Lag, and Lock. These digital control signals indicate whether the DLL is in false-locking state. That is, when the delay of the VCDL is too small, the Lead control signal is activated. The Lag control signal is activated to indicate the delay is too large. The lock detector can take over the loop from PFD and directly sense the charge pump to charge or discharge the loop through the Lead and the Lag control signals. On the other hand, if the delay of the VCDL is within the correct-locking range, the Lock control signal is activated and the DLL core restarts to acquire locking as usual. The auxiliary digital self-correcting loop extends correct-locking range of the DLL four times wider than that of the conventional design constrained by the inequality (1).

The critical path timing contributed by gate delays of the digital decision logics in the auxiliary digital self-correcting loop may be so large in high-speed operation that the switching from the auxiliary digital self-correcting loop to the DLL core cannot be accomplished in time. This might result in instability of the loop. The PFD is adopted to effectively increase switching timing margin and mitigate the problem of loop instability because it has a wider capture range of $(-2 \pi,+2 \pi)$ than the phase detector (PD) used in the previous architecture [5].

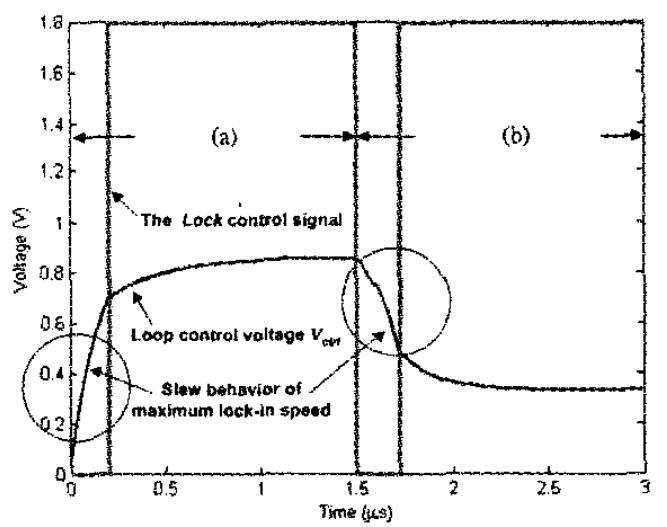

Fig. 2 Simulated loop acquisition behavior of the DLL (a) at $40 \mathrm{MHz}$ and (b) at input clock frequency changed to $100 \mathrm{MHz}$ after $1.5 \mu \mathrm{s}$.

Fig. 2 shows the simulated loop control voltage $V_{c r r l}$ and the Lock control signal when the DLL starts to acquire locking. In Fig. 2(a), the DLL starts at an input clock frequency $40 \mathrm{MHz}$ and the minimum VCDL delay. Once the lock detector detects the DLL core in false-locking state, it immediately disables the Lock control signal. Although the DLL core suffers from false locking, the loop can still lock to the right direction and exhibit slew behavior of maximum speed because of the operation of the auxiliary digital self-correcting loop. The Lock control signal is activated and the DLL core then regains control of the loop until the delay of the VCDL is within the correct-locking range. When the input clock frequency is suddenly changed to $100 \mathrm{MHz}$ after $1.5 \mu \mathrm{s}$, where the conventional DLLs would suffer false- locking problem, the proposed DLL can still react and operate properly, as shown in Fig. 2(b).

\section{Circuit Design}

\section{A. Lock Detector}

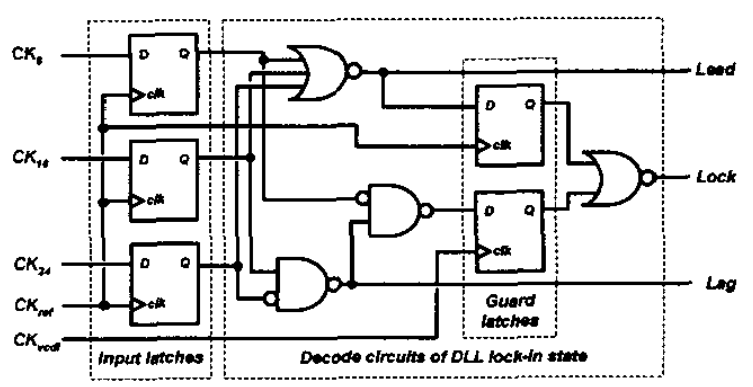

Fig. 3 Schematic of the proposed lock detector.

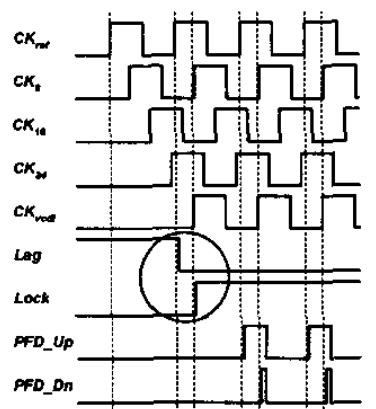

(a)

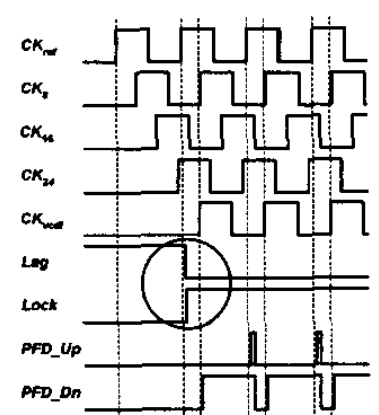

(b)
Fig. 4 Timing diagram of the DLL (a) with and (b) without guard latches included in the lock detector.

The schematic of the lock detector is shown in Fig. 3. The three delayed clocks from the VCDL, $C K_{8}, C K_{16}$, and $C K_{24}$ are first latched at the rising edge of the input reference clock $C K_{\text {ref. }}$ The outputs of these latches are further processed to determine the present lock-in state by decode circuits. Two guard latches, which are trigged respectively by $C K_{r e f}$ and the delayed clock from the last stage of the VCDL, $C K_{\text {ved }}$, are included in the decode circuits generating Lock control signal. Fig. 4 shows the detailed timing diagram of the DLL when the lock-in state of the DLL is changed from the Lag state to the Lock state. It illustrates the functions of the guard latches. The guard latches ensure the Lock control signal can be activated at the right moment so that the PFD can generate proper control signals to keep the DLL core locking to the correct direction when it regains control of the loop from the reset state.

\section{B. Dynamic-Switched Phase-Frequency Detector}

Fig. 5 shows the dynamic-switched PFD circuit, which consists of a dynamic logic PFD and digital control logics for lock detector. The Lead and Lag control signals can bypass the dynamic logic PFD to directly control the following chargepump stage through these digital control logics. The logics included in the reset path properly reset the PFD when the Lock control signal is disabled. The extra gate delays in the reset path also help the PFD periodically produce a chain of short pulses in 
the locked state which can reduce the dead zone of the PFD effectively. The dynamic logic PFD is adopted here for the capability of higher speed operation in comparison with the static counterpart.

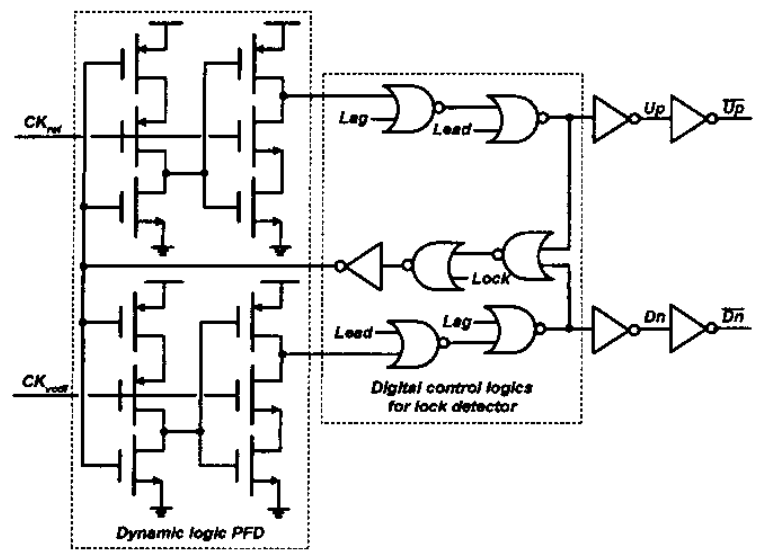

Fig. 5 Schematic of the dynamic-switched PFD circuit

\section{Charge Pump and Voltage-Controlled Delay Line}

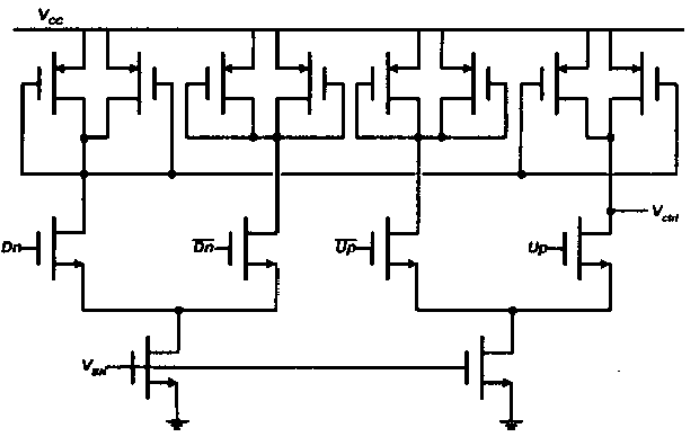

(a)

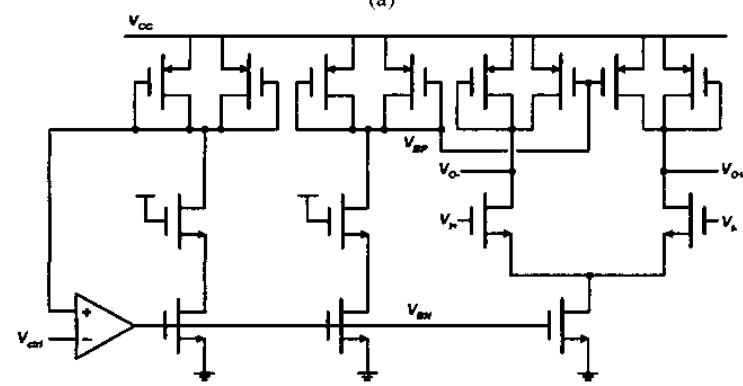

(b)

Fig. 6 (a) Charge pump (b) delay cell of the VCDL with replica bias [6]

The charge-pump circuit and the delay cell of the VCDL with replica bias are similar to those used in [6], as shown in Fig. 6. These circuits are biased with the VCDL control voltage, and their currents can be scaled with the operating frequency of the DLL consequently.

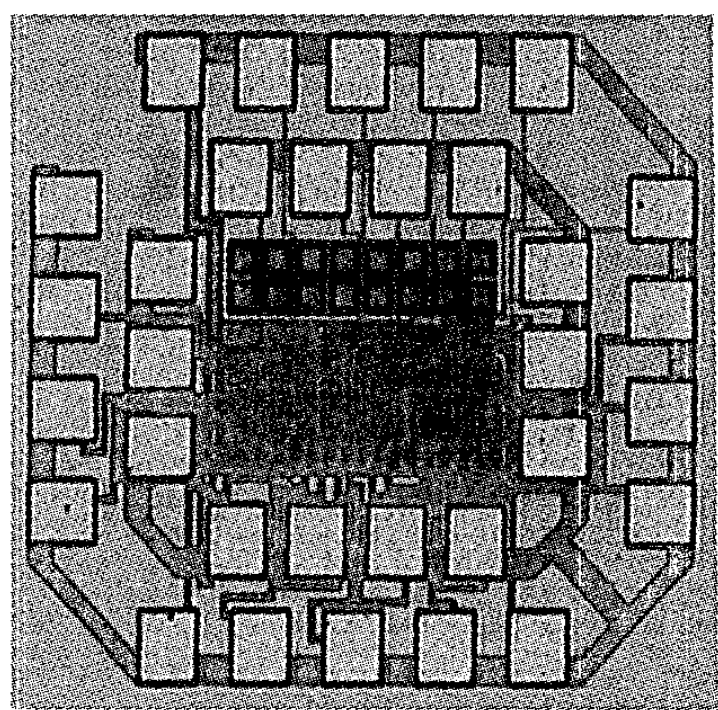

Fig. 7 Die photo of the DLL.

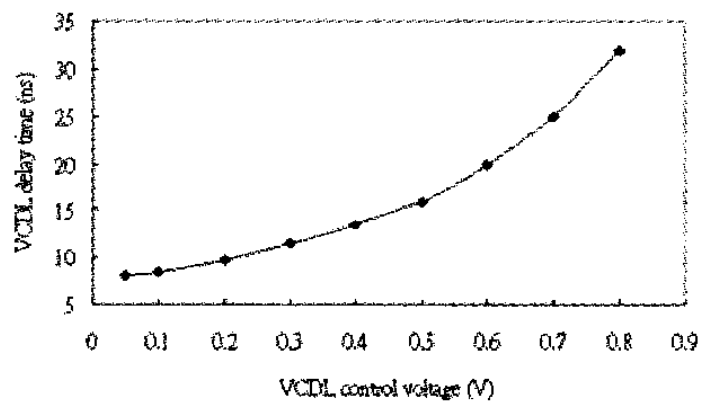

Fig. 8 Measured transfer curve of the VCDL.

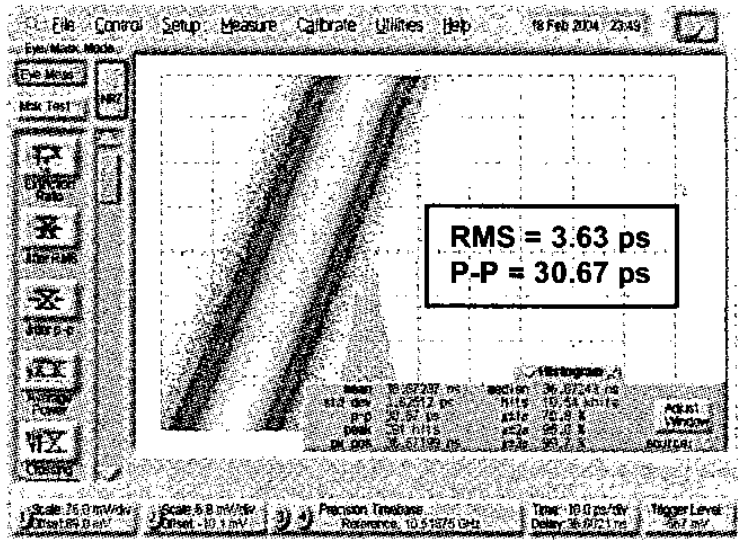

Fig. 9 DLL output jitter histogram at $125 \mathrm{MHz}$.

\section{EXPERIMENTAL RESULTS}

The DLL has been designed and fabricated in a $0.18-\mu \mathrm{m}$ CMOS technology. As shown in the die photo in Fig. 7, the DLL core area is $0.09 \mathrm{~mm}^{2}(360 \mu \mathrm{m} \times 245 \mu \mathrm{m})$. The experimental results show that the DLL can operate in the frequency range of 31.25 to $125 \mathrm{MHz}$ with a $1.8 \mathrm{~V}$ supply. Fig. 8 depicts the measured transfer curve of the VCDL. The tracking jitter 
performance of the DLL output at $125 \mathrm{MHz}$ is demonstrated in Fig. 9. The jitter histogram measures 3.63-ps rms and 30.67-ps peak-to-peak jitter characteristics in a quiet supply. Fig. 10 shows the measured rms jitter characteristics of the DLL over different operating frequencies. If a $100 \mathrm{mV}$ Gaussian random noise is injected externally to the supply, the rms jitter and peak-to-peak jitter are increased to 12.23 ps and $90.7 \mathrm{ps}$ respectively when the DLL operates at $125 \mathrm{MHz}$, as demonstrated in Fig. 11. The equivalent jitter supply sensitivity is less than $0.91 \mathrm{ps} / \mathrm{mV}$. Table I summarizes the performance of the proposed DLL.

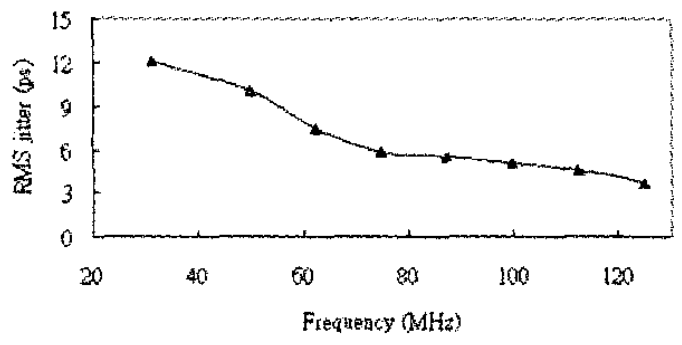

Fig. 10 Measured nns jitter characteristics of the DLL over different operating frequencies.

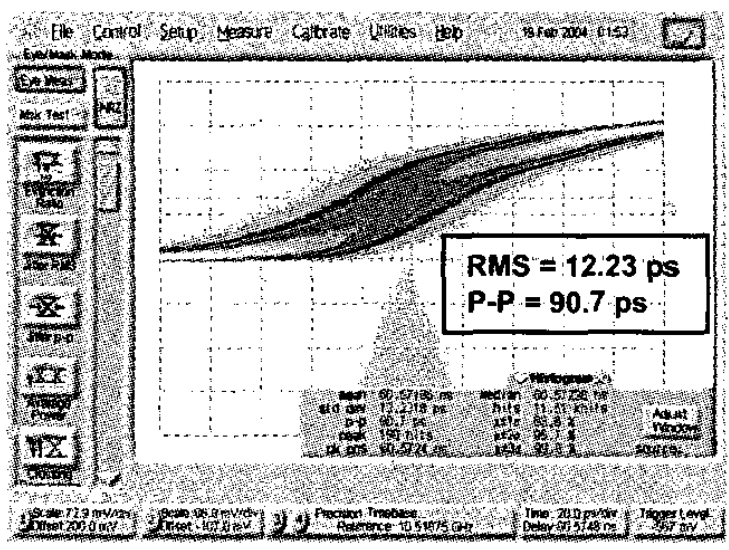

Fig. $11 \mathrm{DLL}$ output jitter histogram at $125 \mathrm{MHz}$ with $100 \mathrm{mV}$ supply noise injected to the supply.

TABLE I

Performance summary of the proposed DLL

\begin{tabular}{c|c}
\hline Process & $0.18-\mu \mathrm{m}$ 1P6M TSMC CMOS process \\
\hline Operating voltage & $1.8 \mathrm{~V}$ \\
\hline Operating frequency range & $31.25 \mathrm{MHz} \sim 125 \mathrm{MHz}$ \\
\hline Output multi-phase clock rate & $1 \mathrm{GHz} \sim 4 \mathrm{GHz}$ \\
\hline RMS jitter & $12.06 \mathrm{ps} @ 31.25 \mathrm{MHz}$ \\
\hline Peak-to-peak jitter & $3.63 \mathrm{ps} @ 125 \mathrm{MHz}$ \\
\hline Supply sensitivity & $88.9 \mathrm{ps} @ 31.25 \mathrm{MHz}$ \\
\hline Lock time & $30.67 \mathrm{ps} @ 125 \mathrm{MHz}$ \\
\hline Power dissipation & $<0.91 \mathrm{ps} / \mathrm{mV}$ \\
\hline Active area & $\sim 54 \mathrm{clock} \mathrm{cycles} \mathrm{(simulated)}$ \\
\hline
\end{tabular}

\section{CONCLUSION}

A self-biased adaptive bandwidth DLL using an auxiliary digital self-correcting loop is proposed to achieve an optimal jitter transfer characteristic and avoid false-locking problem over a wide frequency range and across PVT variations. Compared with previous wide-range DLLs [4]-[8], the proposed DLL demonstrates smaller area cost, lower power consumption, and better jitter performance. The equivalent sampling clock rate of multi-phase clocks generated from 32 taps of the VCDL can operate up to $4 \mathrm{GHz}$, which makes this DLL suitable for lowjitter clock generation in low-band UWB application.

\section{ACKNOWLEDGEMENT}

The authors would like to acknowledge Wei-Hsuan Tu, Hueng-Jie Tsai, Chien-Chih Lin and Prof. Shen-Iuan Liu for valuable technical discussions, Sung-Rung Han, Hsiang-Hui Chang, Rong-Jyi Yang and Chun-Pang Wu for testing support. They would further like to thank National Chip Implementation Center, Hsin Chu, Taiwan, for chip fabrication. This work was supported in part by MediaTek Inc. Hsin Chu, Taiwan 300 .

\section{REFERENCES}

[1] "First report and order in the matter of revision of Part 15 of the commission's rules regarding Ultra-Wideband transmission systems," FCC, released, ET Docket 98-153, FCC 02-48, Apr. 22, 2002.

[2] I. O. Donnell, S. W. Chen, B. T Wang, and R. Broderson, "An integrated low power ultra- wideband transceiver architecture for low rate indoor wireless systems," IEEE CAS Workshop on Wireless Communications and Networking, Sep, 2002.

[3] B. Kim, T. Weigandt, and P. Gray, "PLLDLL system noise analysis for low jitter clock synthesizer design," Proc. Int. Symp. Circuits and Systems, Vol. 4, 1994, pp. 31-38.

[4] Y. Moon, J. Choi, K. Lee, D.-K. Jeong, and M.-K. Kim, "An all-analog multiphase delay-lock loop using a replica delay line for wide-range operation and low-jitter perfornance," IEEE J. Solid-State Circuits, vol. 35, no.3, pp. 377-384, Mar. 2000.

[5] D. J. Foley and M. P. Flynn, "CMOS DLL-based 2-V 3.2-ps jitter 1$\mathrm{GHz}$ clock synthesizer and temperature-compensated tunable oscillator," IEEE J. Solid-State Circuits, vol. 36, no.3, pp. 417-423, Mar. 2001.

[6] J. Maneatis, "Low-jitter process-independent DLL and PLL based on self-biased techniques," IEEE J. Solid-State Circuits, vol. 31, no.11, pp. 1723-1732, Nov. 1996.

[7] S. Sidiropoulos and M. A. Horowitz, "A semi-digital dual delay-locked loop," IEEE J. Solid-State Circuits, vol. 32, no.11, pp. 1683-1692, Nov. 1997.

[8] H. H. Chang, J. W. Lin, C. Y. Yang, and S. I. Liu, "A wide-range delay locked loop with a fixed latency of one clock cycle,"IEEE J. Solid-State Circuits, vol. 37, no.8, pp. 1021-1027, Aug. 1997. 